\title{
MAPEAMENTO DO USO E OCUPAÇÃO DO SOLO NA BACIA DO RIO VERRUGA
} - BA

\author{
Jean de Jesus Novais ${ }^{1}$, Carlos Felipe Oliveira Moura $^{2}$, Safira Rodrigues de Souza ${ }^{3}$, \\ Cassia Juliana Fernandes Torres ${ }^{4}$
}

1. Engํ․ Agrônomo, Mestrando em Agronomia,Universidade de Brasília, Pós-graduação em Geotecnologias: Soluções em Inteligência Geográfica, Escola de Engenharia de Agrimensura.

2. Eng ํㅗㅁônomo, Pós-graduação em Geotecnologias:Soluções em Inteligência Geográfica, Escola de Engenharia de Agrimensura.

3. Eng ${ }^{\circ}$ Agrônoma, Pós-graduação em Geotecnologias:Soluções em Inteligência Geográfica, Escola de Engenharia de Agrimensura.

4. Doutoranda em Energia e Ambiente da Universidade Federal da Bahia, Professora da

Faculdade da Estácio (torres cjf@yahoo.com.br)Salvador-Brasil.

Recebido em: 08/04/2016 - Aprovado em: 30/05/2016 - Publicado em: 20/06/2016 DOI: 10.18677/Enciclopedia_Biosfera_2016_063

\begin{abstract}
RESUMO
O advento das geotecnologias impulsionou o desenvolvimento de estudos socioambientais, uma vez que simplifica e facilita relações entre vários fatores intrínsecos à essa área. Tendo em vista esse aspecto, realizou-se um processamento de dados a partir de Sistemas de Informações Geográficas, a fim de realizar o mapeamento do uso e ocupação de solo na bacia do rio Verruga, Localizado no sudoeste da Bahia, um importante afluente da região que sofre constantes impactos referentes ás atividades antrópicas. Para tanto, foram analisadas imagens orbitais do sensor OLI datadas nos períodos de agosto, setembro e outubro de 2015, com orbitas/pontos 217/070, 216/070 e 216/071, respectivamente. A partir da composição falsa-cor R5B4G3, que realça as feições dos alvos de interesse, foram segmentadas em classes de uso e ocupação do solo. Para a análise da qualidade dos resultados, pontos de controle foram randomizados em uma grade e posteriormente verificados em campo. Com a aplicação do índice Kappa, foi alcançado um valor de 0,73 o que representa a capacidade da metodologia de classificação supervisionada pela técnica de máxima verossimilhança em predizer as características espectrais das classes determinadas, porém, com algumas limitações devido à qualidade dos dados utilizados.
\end{abstract}

PALAVRAS-CHAVE: Bacia Hidrográfica, Planejamento, Sensoriamento Remoto.

\section{USE AND LAND OCCUPATION MAPPING IN VERRUGA RIVER WATERSHED - BA}

\section{ABSTRACT}

The advent of geotechnology boosted the development of social and environmental studies, as it simplifies and facilitates relations between several intrinsic factors to this area. having appearance in view of this, there was hum data processing from Geographic Information Systems, in order to accomplish of use and Occupancy Mapping the Basin of the Verruga River in Southwest Bahia, an important tributary of ENCICLOPÉDIA BIOSFERA, Centro Científico Conhecer - Goiânia, v.13 n.23; p. 7202016 
the Region que suffer constant Impact of anthropogenic activities. Therefore, were analyzed orbital images OLI sensors dated in August September and October 2015 with orbiters / Points 217/070, 216/070 and 216/071. Starting from the false-color composition R5B4G3, that highlights the features of target interest, which were segmented into classes of use and occupation. For the quality results analysis, points were randomized in a grid and later verified in the field. With the application of the Kappa index was reached the value (0.73), What showed a capacity of supervised rating methodology of maximum likelihood technique in predicting how spectral characteristics of each particular class, but with some limitations due to the quality of data used.

KEYWORDS: Hydrographic Basin, Planning, Remote Sensing.

\section{INTRODUÇÃO}

O conhecimento acerca do uso e ocupação do solo pode auxiliar no planejamento socioeconômico e ambiental de uma determinada região (ARAÚJO et al., 2010). Desde o final do século XX, o uso de tecnologias de sensoriamento remoto associado a Sistemas de Informações Geográficas (SIG) tem proporcionado a identificação de terras potencialmente produtivas ou mesmo, as mais suscetíveis à degradação em caráter ambiental (MYERS, 1983). Neste sentido, as geotecnologias assumem suma importância para o desenvolvimento e planejamento de estudos em várias áreas, fato evidenciado em diversos trabalhos sobre sua aplicação em diferentes ramos da ciência, inclusive, envolvendo os aspectos agroambientais (FLORENZANO, 2002; ALMEIDA et al., 2011; DIAS et al. 2013).

Uma Bacia Hidrográfica corresponde ao resultado de ações hidrológicas em torno de cursos d'água (rio principal e afluentes) associada a fatores como geologia, geomorfologia, vegetação e clima (BRIGANTE \& ESPÍNDOLA, 2003). A bacia hidrográfica é a unidade de planejamento e gestão dos recursos hídricos (LEI № 9433/1997), serve de base para as tomadas de decisões no que tange a formulação de políticas públicas, planejamento e de gestão territorial de caráter socioambiental e econômico.

Nos últimos anos, a preocupação com a sustentabilidade, fez crescer a demanda de projetos, planos e estratégias que associem a integração dos diferentes agentes físicos, econômicos e sociais, que atuam no meio, em vista da intensa modificação e degradação ambiental gerada pelo homem (SOFRONIE \& STOICA, 2014). Diante disso, as pesquisas são amparadas pelo uso de técnicas de geotecnologias como ferramentas para o mapeamento temático, análise, avaliação e diagnóstico agroambiental, de impactos e ordenamento territorial entre vários outros fins (MULDER et al., 2011).

Os dados de sensores orbitais retratam as respostas espectrais dos alvos na superfície da terra (JENSEN, 2009). As imagens obtidas a partir do sensor OLI, da plataforma LANDSAT - 8, permitem a combinação de diferentes bandas do espectro eletromagnético a fim de realçar as feições de interesse em uma determinada área, nesse caso específico, a composição R5, G4 e B3, foi escolhida por destacar as feições de solo exposto e vegetação permitindo uma análise mais precisa de seus espectros nas imagens (LILLESAND \& KIEFER, 2000).

Segundo BAIO (1996) às técnicas de classificação digital de imagem permite a elaboração de mapas temáticos nos quais as feições dispostas na superfície são generalizadas segundo seus parâmetros comuns, atribuindo-se a cada pixel uma determinada classe ou categoria predefinida. Diante do exposto, o objetivo do presente trabalho é demonstrar a aplicação de SIG para o mapeamento digital de 
diferentes classes de uso e ocupação do solo, utilizando-se de material cartográfico disponível para subsidiar as pesquisas e os planejamentos de ações nas esferas socioambientais e econômicas relacionadas ao uso e ocupação do solo na bacia do rio Verruga, localizado no Sudoeste da Bahia.

\section{MATERIAL E METODOS}

\section{Área de estudo}

A Bacia Hidrográfica do Rio Verruga compreende os municípios de Vitória da Conquista, Barra do Choça e Itambé, todos localizados no Sudoeste do Estado da Bahia compreendendo as coordenadas UTM, no fuso 24S, 8.360.625S e 332.379E metros (Figura 1). A bacia possui o Noroeste e o Sudeste como direção de fluxo, o qual desagua no Rio Pardo, próximo à cidade em Itambé. Ocupa uma área de aproximadamente $91.136,43$ hectares.

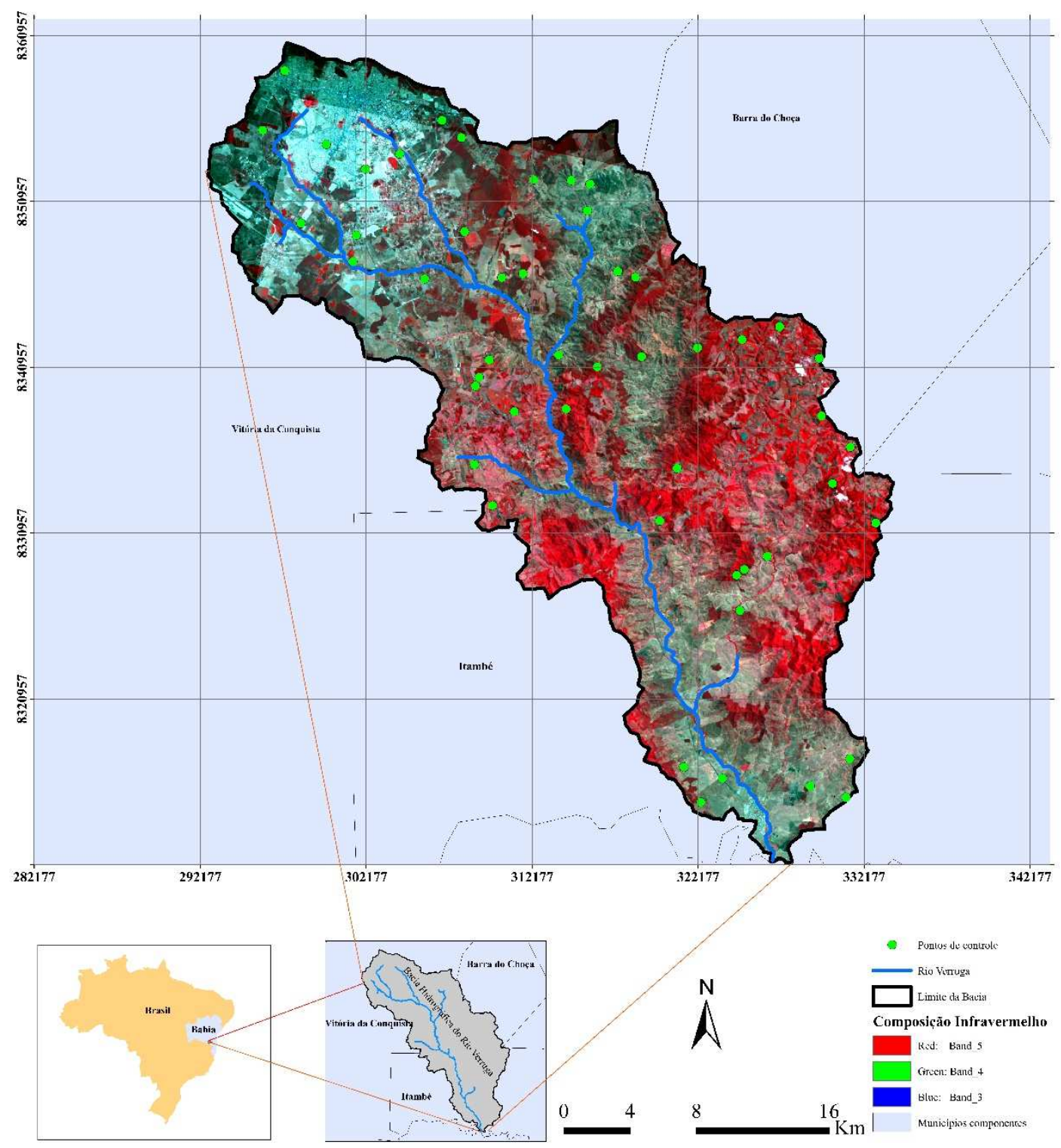

FIGURA 1. Mapa temático da localização da área de estudo contendo os pontos de controle 
O Rio Verruga tem sua nascente primária preservada por uma reserva ecológica conhecida como nascente do Poço Escuro, localizada na Serra do Periperi em Vitória da Conquista. Seus principais afluentes são: o riacho Santa Rita, Córrego Lagoa de Baixo e Rio Periquito. Sua geologia é representada por unidades pré-cambrianas do Complexo Caraíba-Paramirim, e granitoides diversos. As altitudes variam de 700 a $1.100 \mathrm{~m}$. Possui índices pluviométricos variando entre 900 a $1200 \mathrm{~mm}$ anuais, apresentando nos períodos de estiagem um déficit hídrico. A cobertura vegetal é composta basicamente por Floresta Ombófila Densa Submontana, cobrindo $44,7 \%$ do território; a Floresta Estacional Semidecidual com 24\%, a Floresta Estacional decidual com 30,76\% e, 0,54\% do território ocupado com uma vegetação endêmica na Serra do Peri-peri (ROCHA, 2008).

\section{Coleta e tratamento dos dados}

A base de dados teve início com a aquisição do Modelo digital do Terreno (MDT) a partir de arquivos do tipo raster com resolução espacial de 30 metros, obtidos na base nacional de dados do Instituto Brasileiro de Geografia e Estatística (IBGE) relativos à área de estudo as quais pode-se constar as Cartas: SD-24-Y-A; SD-24-Y-B; SD-24-Y-C e D SD-24-Y-D. Com a utilização do Software ArcGis ${ }^{\circledR}$ 10.2.2 (ESRI, 2015), foi gerado um arquivo shapefile, correspondente à área da bacia através da utilização da função Hidrology Tools, que constitui uma extensão desse programa voltado para gerenciamento de dados hidrológicos, onde foram realizados procedimentos para delimitação da bacia hidrográfica a partir do MDT.

Após essa etapa, foram selecionadas e adquiridas imagens no satélite LANDSAT - 8 do sensor multiespectral OLI (Operation Land Imager), com resolução espacial de 30 metros. No sentido de abranger toda a área da bacia houve a necessidade de se obter três imagens do sensor para a montagem do mosaico. Os critérios para obtenção foram: menor percentual de cobertura por nuvens e menor intervalo de tempo entre as cenas (Tabela 1)

TABELA 1. Imagens adquiridas segundo os critérios adotados.

\begin{tabular}{cccc}
\hline Ordem & Órbita/ponto & Data & Ano \\
\hline 1 & $217 / 070$ & 24 de agosto & 2015 \\
2 & $216 / 070$ & 02 de setembro & 2015 \\
3 & $216 / 071$ & 04 de outubro & 2015 \\
\hline
\end{tabular}

O sensor OLI fornece imagens nas bandas: Multiespectral (Bandas 1 a 7 e 9), Pancromática (Banda 8) e Termal (Bandas 10-11), com 30, 15 e 100 metros de resolução espacial, respectivamente. As imagens para esse estudo foram adquiridas a partir da plataforma eletrônica Earth Explorer do site da United States Geological Survey - USDA (ESTADOS UNIDOS, 2015a).

Para o processamento digital das imagens utilizou-se os softwares ENV ${ }^{\circledR}$ (EXCELIS, 2015) e $\operatorname{ArcGis}^{\circledR}$ (ESRI, 2015), nas versões 5.1 e 10.2.2, respectivamente. Deste modo, em decorrência das diferenças de datas e órbitas das imagens utilizadas, os histogramas foram equalizados a fim de uniformizá-los para uma melhor diferenciação dos alvos no momento da classificação. Em seguida, foram realizadas correções radiométrica e atmosférica em cada uma das bandas utilizadas a partir da utilização do algorítimo FLAASH (Fast Line-of-sight Atmospheric Analysis of Spectral Hypercubes). Essas etapas foram desenvolvidas no software $E N{ }^{\circledR}$ (EXCELIS, 2015). Houve então, a importação desses dados para o ArcGis ${ }^{\circledR}$ 
onde, as imagens foram georreferenciadas para o sistema de projeção de coordenadas planas UTM e reprojetadas para o hemisfério sul na zona $24 \mathrm{~S}$, tendo como elipsoide de referência (Datum) Sirgas 2000, para um melhor aproveitamento e controle da qualidade do estudo.

O mosaico composto por seis bandas (bandas de 1 a 6) foi sobreposto à área da bacia, a qual fora previamente delimitada. Fez-se então, uma composição entre as bandas, 5R, 4G, 3B respectivamente, por apresentarem uma maior capacidade na identificação das feições pertinentes ao trabalho, sendo o tipo de uso e ocupação do solo (FLORENZANO, 2002). Inicialmente, foi gerada uma base matricial de Uso do Solo a partir da Classificação Supervisionada pelo algoritmo MaxVer (Máxima Verossimilhança) no mesmo programa através da extensão Spatial Analysis por intermédio da funcionalidade Image Classification do ArcMap ${ }^{\circledR}$ (Aplicação do $\operatorname{ArcGis}^{\circledR}$ ). Foram determinadas seis categorias como apresento no quadro 1.

QUADRO 1 - Descrição das classes a serem delimitadas

\begin{tabular}{|c|c|}
\hline CLASSE & DESCRIÇÃO \\
\hline Área Urbanizada & $\begin{array}{l}\text { Trechos urbanizados, povoamentos e áreas com alta } \\
\text { atividade antrópica. }\end{array}$ \\
\hline Florestamentos & $\begin{array}{l}\text { Matas ciliares e de galeria e às áreas de vegetação natural } \\
\text { mais densa, que correspondem às áreas de reserva ou } \\
\text { remanescentes }\end{array}$ \\
\hline Vegetação Nativa & $\begin{array}{l}\text { Áreas vegetadas que apresentam cor e textura menos } \\
\text { intensa que às áreas anteriores, interligadas a áreas de } \\
\text { pasto (pastagem sombreada ou capoeira) }\end{array}$ \\
\hline Recursos Hídricos & $\begin{array}{l}\text { Áreas que oferecem uma resposta espectral de tonalidades } \\
\text { mais escuras, evidenciando a grande presença de água no } \\
\text { solo. } \\
\text { Áreas de formato na maioria das vezes geométrico e que se }\end{array}$ \\
\hline Solo Exposto/ Agricultura & $\begin{array}{l}\text { encontram sem vegetação, uma vez que a imagem foi obtida } \\
\text { no auge do período seco na região, o que leva a crer que, no } \\
\text { período das águas, estas áreas destinam-se aos cultivos. }\end{array}$ \\
\hline Pastagem & $\begin{array}{l}\text { Áreas de grande abrangência, representada por espaços } \\
\text { com resposta espectral em tons suaves e textura mais lisa }\end{array}$ \\
\hline
\end{tabular}

Fonte: Adaptado de MARTINS (2009)

A classificação constituiu na aquisição de amostras (com média de 1500 pixels) de cada tipo de uso. Para facilitar a compreensão das feições, a imagem foi submetida a uma equalização de histograma, referente a cada banda (R5, G4 e B3). Como resultado, foi elaborada uma base matricial com as classes de uso do solo da área de interesse que, posteriormente, foi convertido em vetor para exportar no formato "shapefile". Ainda com o SIG ArcGis ${ }^{\circledR}$, o mapa obtido foi corrigido e finalizado. $O$ afinamento dos polígonos que ficaram ambíguos foi executado através do "Fild Calculator" para alteração dos atributos e reclassificação respectiva a sua classe correta, gerando o mapa temático final.

A validação dos resultados foi feita, à princípio, pelo confrontamento das observações de 50 pontos de controle levantados de forma sistemática (Figura 5), os quais foram somados a mais 250 pontos obtidos de forma automática e aleatória no ArcGis. Baseados na classificação realizada, esses, foram dispostos em uma malha a partir da ferramenta "create random points" o que resultou em 50 pontos para cada classe. Isso corresponde ao número mínimo de observações para uma boa avaliação estatística da acurácia dos resultados (CONGALTON, 1991). Os 50 
primeiros pontos amostrados foram validados em campo, os demais foram observados com o auxílio do software Google Earth ${ }^{\circledR}$ em laboratório. Os dados foram tabulados em uma matriz de confusão também conhecida como matriz de erro (Tabela 3). Com base nesta matriz foi possível aplicar o cálculo da exatidão global, acurácia do produtor, acurácia do usuário e o cálculo do índice de concordância no ArcMap $^{\circledR}$, distribuídos aleatoriamente seguido de visualização em campo de acordo com CONGALTON (1991). Técnicas discretas multivariadas são apropriadas para o caso de produtos de sensoriamento remoto. Nesse caso foi adotado o índice Kappa $(\square)$ de acordo com a equação 1.

$$
K=\frac{N \sum_{i=1}^{r} x_{i i}-\sum_{i=1}^{r}\left(x_{i+} * x_{+i}\right)}{N^{2}-\sum_{i=1}^{r}\left(x_{i+} * x_{+i}\right)}
$$

eq. 1

Onde, $r$ é o número de linhas na matriz; $x_{i i}$ é o número de observações na linha i coluna i, respectivamente e $N$ é o número total de observações (CONGALTON \& GREEN, 2009).

\section{RESULTADOS E DISCUSSÃO}

O uso de imagens orbitais, como neste caso, do sensor OLI - LANDSAT- 8 representa uma ferramenta de suma importância na realização do trabalho. A aquisição sistemática dos dados dessa natureza permite o mapeamento eficaz da área proposta e a possibilidade de contínuo monitoramento, além de ser uma ferramenta essencial para o planejamento do uso do solo (ESTADOS UNIDOS, 2015b). A análise das informações obtidas possibilitou o cálculo da área de abrangência de cada classe como segue a tabela 2.

TABELA 2. Classes de uso e ocupação do solo.

\begin{tabular}{cccc}
\hline Ordem & Classes & Área (ha) & $\%$ \\
\hline 1 & Área Urbanizada & $8.175,24$ & 8,97 \\
2 & Florestamentos & $8.676,18$ & 9,52 \\
3 & Vegetação Nativa & $26.444,70$ & 29,02 \\
4 & Recursos Hídricos & $1.208,61$ & 1,33 \\
5 & Solo Exposto/ Agricultura & $36.493,02$ & 40,04 \\
6 & Pastagem & $10.138,68$ & 11,12 \\
\hline Total & - & $91.136,43$ & 100,00 \\
\hline
\end{tabular}

A partir da Tabela 2, observa-se que a área de vegetação nativa concentradas nos limites das Áreas de Proteção Permanentes (APP) ocupa aproximadamente $29 \%$ da área da bacia, sendo encontrada de forma dispersa e mal distribuída. Na parte norte da bacia encontram-se nascentes primárias, que se apresentam conservadas no perímetro urbano do município de Vitória da Conquista. No curso do rio Verruga, juntamente com seus segmentos, podem ser facilmente observadas às ações antrópicas.

ARAÚJO et al. (2010), aponta alguns reflexos da ação humana sobre o meio, tais como: destruição da vegetação natural, erosão, acumulação da produção de resíduos altamente poluentes, entre outras; destacando ainda que a ocupação indiscriminada dos espaços naturais coloca em risco a segurança e a saúde do próprio homem os quais pode-se citar inundações e escorregamentos de encostas. 
Esses impactos podem facilmente ser notados na bacia do rio Verruga (BONFIM et al., 2012).

Ao longo do curso, o rio Verruga tem como trajetória propriedades urbanas e rurais, além de rodovias e estradas vicinais dos dois municípios os quais compõem a bacia. Grande parte das áreas de preservação permanente encontram-se deteriorada em relação as suas margens, apresentando em seu leito, áreas com avançado estado de poluição. Por se encontrarem desprotegidas, essas margens apresentam uma maior vulnerabilidade à poluição e degradação. ROCHA (2008) ressalta que as águas do rio Verruga encontram-se sob forte pressão da urbanização justamente por deter suas principais nascentes dentro do perímetro urbano de Vitoria da Conquista, mesmo protegidas por áreas de preservação ambiental.

A Figura 2 apresenta o mapa final de uso e ocupação do solo da bacia do rio Verruga.

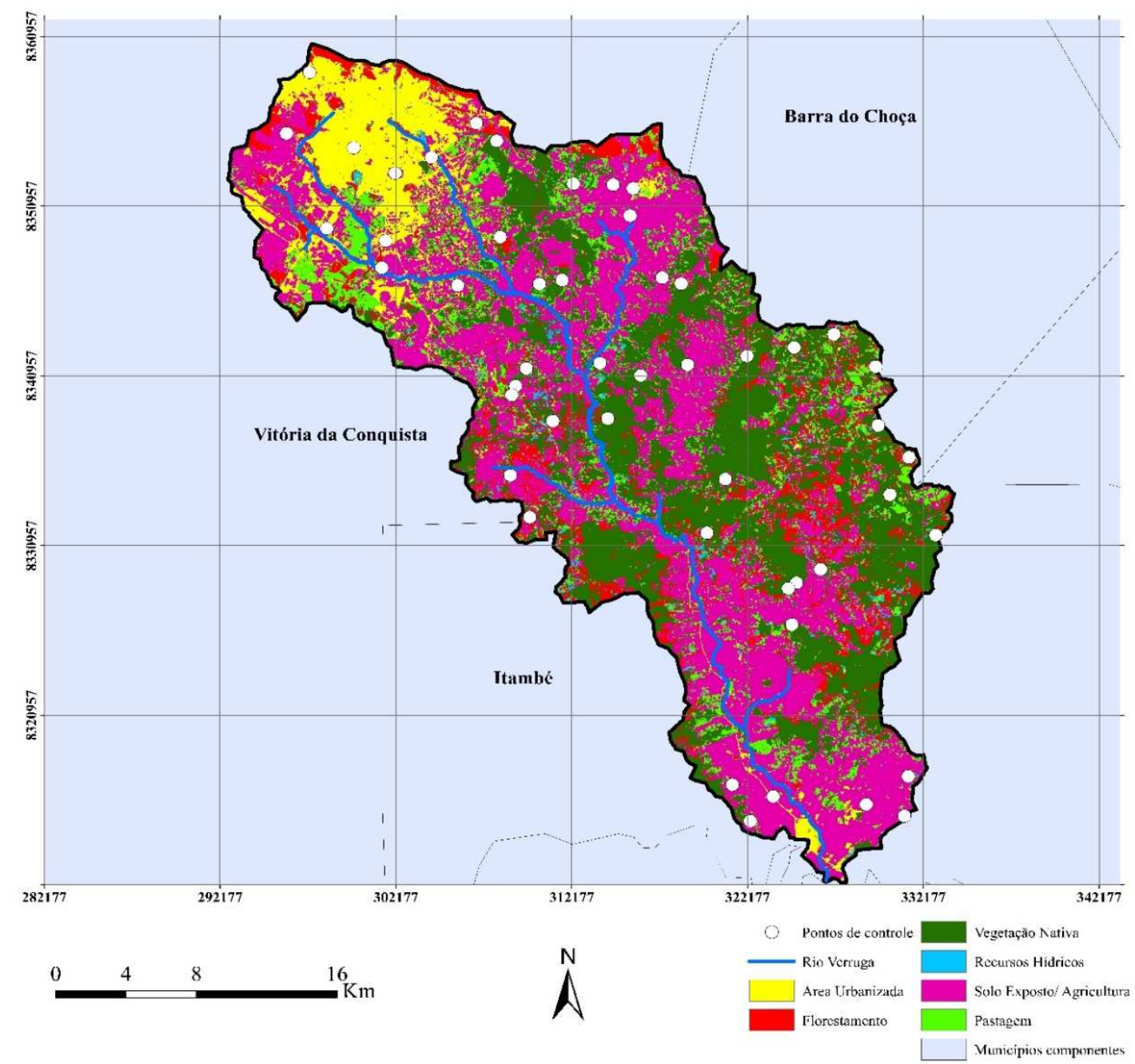

FIGURA 2. Mapa Temático de classe de uso e ocupação do solo na Bacia do Rio Verruga - BA com pontos de controle. 
Com base na Tabela 2 e na Figura 2, observa-se que a combinação de solo exposto e agricultura teve uma grande representação na área da bacia (40\%). Esse valor pode ser justificado, entre outras causas, em virtude das imagens de satélite utilizado neste trabalho ter sido obtida no período de estiagem (Julho a Outubro) onde há uma diminuição da cobertura vegetal nativa e restrição das atividades agrícolas devido, principalmente, aos baixos índices pluviométricos. As pastagens corresponderam mais de 10.000 hectares, a técnica de classificação proporcionou aos resultados obtidos a discriminação das diferentes classes deter áreas de pastagem sombreada ou nativa, natural e implantadas, ambas apresentaram altos índices de correlação. A Tabela 3 apresenta a matriz de confusão da classificação conduzida neste trabalho.

TABELA 3. Matriz de confusão da classificação executada

\begin{tabular}{cccccccc}
\hline Classes & I & II & III & IV & V & VI & $\begin{array}{c}\text { Total da } \\
\text { linha }\end{array}$ \\
\hline I & 44 & 3 & 4 & 1 & 5 & 4 & 61 \\
II & 3 & 27 & 5 & 2 & 3 & 4 & 44 \\
III & 3 & 3 & 31 & 1 & 3 & 6 & 47 \\
IV & 1 & 0 & 1 & 39 & 1 & 0 & 42 \\
V & 4 & 2 & 3 & 1 & 45 & 6 & 61 \\
VI & 3 & 1 & 2 & 2 & 4 & 33 & 45 \\
\hline $\begin{array}{c}\text { Total da } \\
\text { Coluna }\end{array}$ & 58 & 36 & 46 & 46 & 61 & 53 & $\mathbf{3 0 0}$ \\
\hline
\end{tabular}

Onde, I - Área Urbanizada; II - Florestamentos; III - Vegetação Nativa; IV - Recursos Hídricos; V - Solo Exposto/ Agricultura; VI - Pastagem

Do total de pontos amostrados, 219 corresponderam ao real, onde a maioria se encontraram incluídos nas classes I - Área Urbanizada e IV - Recursos Hídricos, por outro lado, os pontos de controle relativos às áreas de vegetação nativa, florestamentos e solo exposto/ agricultura apresentaram a maior parte das discrepâncias da realidade em campo. Fato justificado pelo fato dessas atividades estarem sujeitas aos fatores climáticos.

A exatidão global corresponde à estatística descritiva desse tipo de análise, é obtida dividindo-se o somatório da diagonal principal (Tabela 4, destaque em cinza) pelo número total de pontos amostrados (Tabela 4, número em negrito) $(219 / 300=$ 73\%). Seguindo essa lógica, divide-se o número de pontos acertados em dada categoria pelo total amostrado em cada linha ou coluna da matriz de confusão para se obter a acurácia da classificação. O resultado dessas operações, em nível de coluna (acurácia do produtor), indica a probabilidade de um ponto de referência da classe ter sido classificado da maneira correta, isto denota uma medida de erro de omissão (Tabela 5). Entretanto, se o número total de acertos em uma categoria é dividido pelo número total de pontos classificados nessa categoria, então, esse resultado é uma medida de erro de comissão (acurácia do usuário ou confiabilidade), o que indica que um ponto classificado no mapa/imagem realmente representa essa categoria na realidade de campo (CONGALTON \& GREEN, 2009). Os resultados podem ser observados na Tabela 4. 
TABELA 4 - Tabela analítica de acurácia da matriz de confusão

\begin{tabular}{cccccc}
\hline \multicolumn{7}{c}{ Exatidão Global (\%) } \\
\hline \multicolumn{7}{c}{$219 / 300=73,00$} \\
\hline \multicolumn{7}{c}{ Acurácia do Produtor (\%) } \\
\hline I & II & III & IV & V & VI \\
\hline 72,13 & 61,36 & 65,96 & 92,85 & 73,77 & 73,33 \\
$44 / 61$ & $27 / 61$ & $31 / 47$ & $39 / 42$ & $45 / 61$ & $33 / 45$ \\
\hline \multicolumn{7}{c}{ Acurácia do Usuário (\%) } \\
\hline 75,86 & 75,00 & 67,39 & 84,78 & 73,77 & 62,26 \\
$44 / 58$ & $27 / 36$ & $31 / 46$ & $39 / 46$ & $45 / 61$ & $33 / 53$ \\
\hline
\end{tabular}

Analisando a tabela 4, a estatística descritiva da exatidão global atingiu a proporção de $73 \%$ de exatidão, é possível observar que a classe IV - Recursos Hídricos, foi a que alcançou maior probabilidade de acertos na classificação tanto acurácia do produtor (92,85\%), quanto na acurácia do usuário (84,78\%), o que é considerada como boa a muito boa (CONGALTON \& GREEN, 2009), Isso se dá pelo fato de ser uma classe totalmente distinta das demais, dada a alta taxa de absorção das ondas eletromagnéticas exercida pela água (JENSEN, 2009). Os menores percentuais de acurácia ocorreram na classe de vegetação nativa, pois, essa possui valores de pixels distribuídos em todas as demais classes.

Para a estimativa da acurácia, os resultados foram submetidos à análise do Índice de Kappa, onde inclui o uso de todos os elementos da matriz de confusão. Esse Índice varia de 0 a 1, onde quanto mais próximo de 1 melhor a precisão. 0 valor calculado foi de $0,73 \%$, que segundo CONGALTON \& GREEN (2009) é considerado muito boa.

\section{CONCLUSÕES}

O mapeamento das classes de uso e ocupação do solo da bacia do rio Verruga utilizando o software ArcGis pelo método de Máxima verossimilhança se mostrou satisfatório, levando em consideração o fato desse software não ser especificamente voltado para processamento digital de imagem, a técnica apresentou uma boa qualidade nos resultados. Contudo, a intercalação de métodos e ferramentas relacionadas a este fim, pode melhorá-los consideravelmente adotando-se procedimentos de correção de imagem, por exemplo. Constatou-se que - maior percentual da área estudada corresponde às feições relacionadas a agricultura e solo exposto. No que se refere ao objetivo do trabalho, a análise se mostrou capaz para auxiliar nas tomadas de decisão no que se refere aos aspectos socioambientais e econômicos na bacia do rio Verruga.

\section{AGRADECIMENTOS}

Ao Instituto de Qualificação Profissional - IQUALI pelo auxílio no desenvolvimento deste estudo.

\section{REFERÊNCIAS}

ALMEIDA, A. S.; SANTOS, R. L.; CHAVES J. M. Mapeamento de Uso e Ocupação do Solo no Município de Jeremoabo-Ba: Uso do Algoritmo Máxima Verossimilhança (Maxver). Anais: XV Simpósio Brasileiro de Sensoriamento Remoto - SBSR, p. 7255-7262, 2011.

ARAÚJO, G. H. de S.; ALMEIDA, J. R. de; GUERRA, A. J. T. Gestão ambiental de áreas degradadas. 5\%. ed. rev., Rio de Janeiro: Bertrand Brasil, 322 p., 2010. 
BAIO, M. A detecção remota como fonte de informação geográfica. 1996. 151 f. Dissertação (Mestrado em Ciências e Sistemas de Informação Geográfica) Universidade Nova de Lisboa, Instituto Superior de Estatística e Gestão de Informação.

BONFIM, D. A.; SANTOS, J. O.; SAMPAIO, R. J.; SILVA JUNIOR, M. F. Considerações sobre as mudanças climáticas e os impactos na sub-bacia do rio Catolé para o município de Vitória da Conquista, Bahia. Rev. Eletrônica do Mestrado Em Educação Ambiental, v. 29, p. 1 - 16, 2012.

BRIGANTE, J.; ESPÍNDOLA, E. L. G. Limnologia Fluvial: Um Estudo no Rio Mogi-Guaçu. São Carlos: Editora RiMa, 278p, 2003.

CONGALTON, R.G. A Review of Assessing the Accuracy of Classification Remotely Sensed Data. Remote Sensing of Environment, v. 37, p. 35-46, 1991. DOI: 10.1016/0034-4257(91)90048-B.

CONGALTON, R.G.; GREEN, K. Assessing the accuracy of remotely sensed data: principles and practices. Principles and Practices. New York: Lewis Publishers, 2 ed., 179p, 2009.

DIAS, L. F.; LOBÃO, J. S. B.; MACHADO R. A. S. Mapeamento do uso e ocupação do solo do município de Feira de Santana, Bahia. Anais:XVI Simpósio Brasileiro de Sensoriamento Remoto - SBSR, p.7398-7405, 2013.

ESRI - Enviromental Systems Research Institute, Inc. ArcView Manual. 2015. Disponível em: <http://www.esri.com/software/arcview/about/literature.html>.

ESTADOS UNIDOS. United States Geological Survey. 2015a. Earth Explorer. Disponível em: <http://earthexplorer.usgs.gov/>.

ESTADOS UNIDOS. United States Geological Survey. 2015b. LandSat Missions. Disponível em: < http://landsat.usgs.gov/>.

FLORENZANO, T. G. Imagens de satélite para estudos ambientais. São Paulo, Ed. Oficina de Textos, 1 ed., 2002.

JENSEN, J.R. Sensoriamento Remoto do Ambiente: Uma Perspectiva em Recursos Terrestres. Tradução português, 2 ed., Parentese Editora, São José dos Campos SP, 598p. 2009.

LILLESAND, T.; KIEFER, R. Remote sensing and image interpretation. New York: John Wiley \& sons, 724p, 2000.

MARTINS, A. P. Bacia do ribeirão cachoeira de cima, Itapagipe (MG): Avaliação ambiental integrada e alternativas de uso. 2009. 227f. Dissertação (Mestrado em Geografia). Universidade Federal de Uberlândia. Geografia e Gestão do Território.

MULDER, V. L.; BRUIN, S.; SCHAEPMAN, M. E.; MAYR, T. R. The use of remote sensing in soil and terrain mapping - A review. Geoderma, v. 162, p. 1-19, 2011. DOI:10.1016/j.geoderma.2010.12.018. 
MYERS, V. I. Remote sensing applications in agriculture. American Society of Photogrammetry. Manual of remote sensing. 2. ed. Fall Church. Cap. 33, p.2119 2136, 1983.

ROCHA, A. A. Análise socioambiental da bacia do Rio Verruga e os processos de urbanização de Vitória da Conquista-Ba. 2008. 179f. Dissertação (Mestrado em Geografia). Universidade Federal da Paraíba. Centro de Ciências Exatas e da Natureza-CCEN.

SOFRONIE, C.; STOICA, F. S. Principles of integrated management of water resources within a hydrographic basin. application in some $\square$ - tisa hydrographic basin. 2nd International Conference - Water resources and wetlands, Tulcea, Romania, p. 1-6, 2014. 